\title{
El feminismo en la novela juvenil actual: Sweet Temptation - Ein Milliardär zum Anbeißen. La evolución de las figuras femeninas en la cultura actual
}

\author{
Irene Pérez Torres \\ Universidad de Sevilla \\ irenesprungli@gmail.com \\ https://dx.doi.org.10.12795/futhark.2019.il4.08
}

Fecha de recepción: 15.10 .2018

Fecha de aceptación: 25.11 .2018

Resumen: A lo largo de la historia, la cultura ha mostrado, inevitablemente, una base sociológica sexista en la que la mujer sirve de objeto de consumo al servicio del hombre, sin el cual se ve perdida en este mundo. Es innegable que el sexismo se combate desde la ideología: si se inculca una educación feminista desde las edades más tempranas, estas ideas de igualdad calarán en mayor medida y darán sus frutos en las futuras generaciones. Es por ello que, con la creciente concienciación feminista en la sociedad, cada vez son más las historias -ya sean novelas, películas, canciones... -en las que la mujer toma el poder de su vida y decide sobre su propio destino, deshaciéndose del lastre tradicional de depender de una figura masculina. Aquí entra en juego la alemana Lotte Römer: en su novela Sweet Temptation - Ein Milliardär zum Anbeißen nos muestra a una joven cuya perspectiva de futuro no se nublará ante los encantos de un millonario. Emilia, la protagonista, decide su propio destino y trabaja para conseguir su sueño sin reclamar ayuda de nadie; sólo de ella misma. Un mensaje de empoderamiento que el público preadolescente al que va dirigido debería conocer, compartir y poner en práctica.

Palabras clave: feminismo, empoderamiento, amor romántico, dependencia emocional, rol de género.

The Feminist Movement in the Teenage Novel Sweet Temptation - Ein Milliardär zum Ambeißen. The evolution of the female role in today's culture 
Abstract: In the course of history, culture has made record of the sexist sociologic base in which women must serve as consume objects for men. Without a masculine figure, women feel lost in this world, having no purpose in their lives other than finding a husband, having children and staying at home making dinner. It is undeniable that sexism must be battled starting from our ideology: if we instill feminism from an early age, it will have an impact in future generations. That is why, as the need of equality makes a mark in society, the number of stories -no matter if they are told in books, films, songs... -in which women take control of their lives, getting rid of emotional dependency, is growing uncontrollably. Here is where German writer Lotte Römer comes into play: in her novel Sweet Temptation - Ein Milliardär zum Anbeißen, she shows us a young woman whose future perspective will not be erased by a charming millionaire man. She not only does not ask any man for help, but also decides on her own destiny and works hard for her "Pastry Passion" to come true: she relies exclusively on herself, and that is more than enough. A message of empowerment that the pre-adolescent reader should know, share and put into practice.

Keywords: feminism, empowerment, romantic love, emotional dependency, gender role.

El presente trabajo científico indaga en las cuestiones feministas incluidas en la novela Sweet Temptation - Ein Milliardär zum Anbeißen, de Lotte Römer, estableciendo una comparación paralela con los crecientes mensajes de empoderamiento' e igualdad latentes en la cultura de hoy: arte, cine $y$, por supuesto, literatura. Para ello, centraremos nuestra atención en la ruptura de clichés sexistas a los que el joven consumidor de novela romántica habitual $-y$, lamentablemente, los niños- suele estar acostumbrado, de modo que observaremos cómo esta nueva oleada de cultura feminista cala en las permeables mentes de los más jóvenes.

A través de un acto tan revolucionario como representa la literatura, Lotte Römer indaga en cuestiones que calan en la permeable mente del público adolescente-juvenil. Dada la premisa chica-conoce-chica que sigue la novela, podría parecer que Sweet Temptation - Ein Milliardär zum Anbeißen no es más que otra novela romántica para preadolescentes deseosas de vivir a través de las páginas de

1 "Por empoderamiento de las mujeres nos referimos al proceso por el cual las mujeres, en un contexto en el que están en desventaja por las barreras estructurales de género, adquieren o refuerzan sus capacidades, estrategias y protagonismo, tanto en el plano individual como colectivo, para alcanzar una vida autónoma en la que puedan participar, en términos de igualdad, en el acceso a los recursos, al reconocimiento y a la toma de decisiones en todas las esferas de la vida personal y social." (CCOO $2017)$. 
un libro una historia de amor de cuento de hadas. Nada más lejos de la realidad. A diferencia del "vivieron felices y comieron perdices", que suele llevar intrínseco como subtexto la dependencia emocional ${ }^{2}$ de una joven hacia el primer hombre que la "salva", Sweet Temptation - Ein Milliardär zum Anbeißen desecha el típico relato de amor romántico ${ }^{3}$, plagando sus líneas de notas sobre feminismo ${ }^{4}$, actualidad social, la brecha salarial de género $0^{5}$ otros puntos que no hacen sino desechar la imagen del príncipe azul protector de una chica desvalida ${ }^{6}$, que jamás podría sobrevivir ni ser feliz sin un hombre que la mantenga. Afortunadamente, parece ser que la cultura en general está empezando a crear conciencia del problema desde las historias de niños, que contienen un mensaje cada vez menos sexista:

2 "Se llama así al sometimiento o sumisión psicológica a una o varias personas, aunque como resultado de esta sumisión se produzca un daño para la persona sometida. La dependencia emocional es propia de las personalidades dependientes." (ADAMED s.f.)

3 “Diana Maffía, filósofa feminista, lo explica así: "El amor romántico es un invento entre el renacimiento y la modernidad que tiene que ver con fijar determinado tipo de roles a partir del cambio en la familia. La idea del amor, la sexualidad y el matrimonio unidos en un mismo espacio es absolutamente reciente. [...] El amor romántico es pensar en dos imanes que en algún momento conforman una totalidad, un amor heterosexual en el cual un varón y una mujer se van a ver atraídos y complementados."

Esto resulta en que una mujer está incompleta hasta que encuentra esa pareja: el todo. También resulta en que a una sola persona se le demanda: exclusividad sexual, amigos en común, hijos, vacaciones y momentos de ocio compartidos. Si este combo no se arma hay vacío y sufrimiento. El amor romántico se adapta bien a una sociedad capitalista en la que prima el individualismo porque excluye otro tipo de alianzas fuertes de afecto." (Gualano 2018)

${ }^{4}$,Die Stichtworte <Frauenbewegung> und <Feminismus> stehen für ein gemeinsames Ziel. In beiden Fällen geht es darum, Frauen in allen Lebensbereichen, in Staat, Gesellschaft und Kultur und vor allem auch in der Privatsphäre, gleiche Rechte und Freiheiten sowie gleiche Teilhabe an politischer Macht und gesellschaftlichen Ressourcen zu verschaffen.“ (Gerhard 2009, 6)

5 „Der sogenannte Gender Pay Gap beschreibt den prozentualen Unterschied zwischen dem durchschnittlichen Bruttostundenverdienst von angestellten Männern und Frauen. Seine Berechnung ist in der Europäischen Union einheitlich geregelt und ist somit europaweit der Hauptindikator für die ungleiche Entlohnung von Männern und Frauen. [...] Der unbereinigte Gender Pay Gap liegt in Deutschland bei 21 Prozent." (Reisin 2018)

6 "The original stories we know as fairytales were made with a purpose: for educating and putting forth ideal behaviours for children, both boys and girls. Boys were taught to be action-takers, leaders, and protectors, often striding forth to either save or find their mysterious princesses, and take their happilyever-after. While the male hero isn't expected to be all that eloquent with his words, he's still expected to be the one to take charge in any situation. Girls got a very different message. In order for them to succeed, they needed to fit into the narrow view of what was beautiful, as well as being kind, generous, self-sacrificing, the epitome of patience and forgiveness - just to name a few. Essentially, they were taught to be as passive and as gentle as possible." (Antebellum 2016) 
In the last decade, Walt Disney Productions has produced several new Disney Princesses for both children and adults to enjoy. "Tangled," "Brave," "Frozen" and "Moana"" all feature stronger, more independent heroines, marking a distinct break in tradition from the damsels-in-distress of the Disney classics. [...] From Walt Disney's first film, "Snow White and the Seven Dwarves," to the most recent production, "Moana," princesses have gradually become more independent. Indeed, these new Disney princesses are fully capable of saving themselves and others. The new films look starkly different from their firstgeneration ancestors, many of which portrayed the Disney princesses as dependent on men. (Batt 2018)

Los tiempos están cambiando y, con ellos, la mentalidad general también progresa: desde las edades más tempranas, Disney hace un trabajo maestro al desechar los anticuados roles de género tradicionales ${ }^{8}$. Como en Sweet Temptation - Ein Milliardär zum Anbeißen, las princesas Disney de "have not only shed their damsel attitudes, which involves waiting for someone to save them, but have become the heroes of their own story and have found a way to save themselves". (Batt 2018). Ahora, las chicas ya no son pobres princesas necesitadas: son dueñas de su propio destino, artífices de su futuro como individuo autosuficiente.

El mensaje evoluciona a la novela juvenil: tanto en el texto de Römer en particular como en la actualidad en general, está claro que el empoderamiento femenino posee una relevancia indiscutible. Con cada caso nuevo de sexismo, con cada acoso, con cada situación de desigualdad, cada día son más las mujeres que se

7"Out of all the recent Disney movies, "Moana" stands alone because there is no mention of a romantic relationship. Moana is on a quest to explore the ocean and save her people; there is no prince in the movie, Charming or otherwise. Even Merida had the opportunity if she wanted it, but Moana doesn't even have that. She doesn't need the distraction of a relationship when her people are at stake." (Batt 2018)

8 „Geschlechterstereotype sind kognitive Strukturen, die sozial geteiltes Wissen über die charakteristischen Merkmale von Frauen und Männern enthalten (Ashmore/Del Boca 1979, Eckes 1997). [...] Für Geschlechterstereotype ist [...] kennzeichnend, dass sie deskriptive und präskriptive Anteile haben. Die deskriptiven Anteile umfassen traditionelle Annahmen darüber, wie Frauen und Männer sind, welche Eigenschaften sie haben und wie sie sich verhalten. Frauen „sind“ danach verständnisvoll und emotional, Männer ,sind“ dominant und zielstrebig. Aus Verletzungen dieser Annahmen folgt typischerweise Überraschung. Die präskriptiven Anteile beziehen sich auf traditionelle Annahmen darüber, wie Frauen und Männer sein sollen oder wie sie sich verhalten sollen. So ,sollen“ Frauen einfühlsam sein, Männer „sollen“ dominieren. [...] Eng verwandt ist das Konzept der Geschlechterrolle. Sein Gebrauch ist allerdings in der Literatur etwas uneinheitlich. Teils werden Geschlechterrollen präskriptiv im Unterschied zu bloß deskriptiv verstandenen Geschlechterstereotypen konzipiert (Alfermann 1996), teils werden sie, insbesondere im Kontext der Analyse von Familien-, Berufs- und Führungsrollen, sowohl in ihren deskriptiven als auch in ihren präskriptiven Funktionen diskutiert (Eagly/Karau 2002, Eagly/Wood/Diekman 2000). In jedem Falle aber liegt die Betonung beim Geschlechterrollenkonzept auf den sozial geteilten Verhaltenserwartungen, die sich auf Individuen aufgrund ihres sozial zugeschriebenen Geschlechts richten.“ (Eckes 2008, I) 
lanzan a escribir literatura en mayúsculas con el objetivo de acabar con los comportamientos y, sobre todo, con las mentalidades machistas. Aquí es donde entra en juego la literatura femenina y feminista. Estos nuevos relatos son indiscutiblemente necesarios hoy, siendo de gran importancia su asequibilidad para las mentalidades más jóvenes y permeables. Ejemplo de ello es también la estadounidense Meg Wolitzer, cuya novela The Female Persuasion ${ }^{9}$ incide también, como Römer, en el mensaje de poder femenino:

Der Roman stellt die Frage, wie Menschen und insbesondere Frauen zu Macht kommen. Sind sie, wenn sie zu Macht gekommen sind, genauso verführbar wie machtvolle Männer, also kurz: Verfügen sie über das gleiche ArschlochPotenzial wie machtvolle Männer? Muss man als Frau männliche Strategien übernehmen, um zu Macht zu kommen? Diese Fragen wiederum werden in eine Zeit hinein formuliert, die unter dem Eindruck des \#MeToo-Erdbebens steht, also jener weltweiten Debatte, die im vergangenen Oktober begann und bis heute andauert und deren Ausgangspunkt sexualisierte Gewalt war, die von mächtigen Männern ausging. (Baum 2018)

Si bien aún vivimos en un mundo desigual en el que, tristemente, las mujeres deben trabajar el doble que los hombres por menos, Wolitzer, Römer, Disney y toda la nueva y creciente corriente feminista intenta que el lector se despoje de esos esquemas tan arraigados en la sociedad actual como necesarios de suprimir.

Frauen, schreibt Wolitzer, seien nicht nur als Rezensentinnen in den "prestigeträchtigen Publikationen" unterrepräsentiert, sie seien auch für den Kreis derjenigen Autoren nicht vorgesehen, die für die wirklich wichtigen Weltbeschreibungsbücher zuständig seien (wie etwa Jonathan Franzen, David F. Wallace). (Baum 2018)

Sweet Temptation - Ein Milliardär zum Anbeißen narra la historia de Emilia, una chica italiana residente en Nueva York, que está al frente de una pequeña tienda de Pasteles, „Pastry Passion“. A lo largo de las páginas vivirá una historia de amor que, sin embargo, no queda en un segundo plano para ella: sigue siendo su prioridad, su sueño y su decisión. A pesar de no ganar una fortuna -criticando, nuevamente, la brecha salarial-, se dedica a su pasión, mensaje que cala desde el primer instante en el lector: „Aber bisher kam sie mit „Pastry Passion“ gut über die Runden. Sie verdiente kein Vermögen, doch sie liebte ihren Job und es reichte zum Überleben.“ (Römer 2016). Como en el caso de The Female Persuasion, donde la protagonista "is the living embodiment of today's growing number of young white women whose

9"The Female Persuasion is about graduating from college and finding one's way in the world, about renegotiating youthful friendships and romantic relationships as an adult, about power and betrayal. It's also about reckoning with the successes and failures of the women's movement and learning how to be a feminist now, in the 2 Ist century, when the old barriers to women's success have been broken down but no one understands quite what has replaced them." (Franklin 2018) 
own processes of politicization are equally inspiring and problematic", la escritora alemana defiende luchar por las pasiones personales frente al capitalismo de personas que se pasan las horas contando los días que restan para el fin de semana, para las vacaciones, para la jubilación. El dinero mueve el mundo en este mundo globalizado, pero nuestra protagonista, Emilia, representa a una revolucionaria modelo a seguir, una feminista que se rebela contra las imposiciones socioeconómicas del patriarcado. Precisamente será este romanticismo ${ }^{10}$ (en el sentido original de la palabra), intrínseco en su carácter, el que le hará llevar su humildad a extremos en los que regala restos de sus pasteles a clientes habituales -como Mrs. Witherleaf- que corresponden con igual amabilidad: „Man muss hier in der Nachbarschaft doch zusammenhalten". Tanto es así, que la protagonista pondrá su solidaridad por encima de sus necesidades: „Natürlich verdiente sie an ihr nichts, aber das war egal“.

Emilia representa un ejemplo de bondad en un mundo cada vez más deshumanizado, un atisbo de esperanza para el cambio. El hecho de que sólo unos pocos clientes se parezcan a Mrs. Witherleaf (,Es gab zum Glück noch ein paar andere Kunden, die das ausgleichen"), constituye una crítica a la vertiginosa sociedad moderna, un rechazo a la velocidad y a la precipitación, por lo que la contextualización de la historia en la ciudad de Nueva York, élite del capitalismo, resulta un golpe maestro. El personaje de la clienta no es en absoluto casual, sino que simboliza la importancia de relacionarse con personas, y no solo con seres que ofrecen dinero a cambio de un producto, la defensa de la humanidad frente a la comercialización de las personas. Además, en su primer encuentro con los empresarios millonarios, éstos se comportan de manera paternalista y ruda, casi sin respeto, dando ejemplo de prácticas como el mansplaining" $\mathrm{y}$ otras actitudes que, al lector de hoy, le resultan despreciables:

El machismo, como el racismo, ha ido mutando. En el mundo occidental y
desarrollado ya no es usual ese sexismo a voces que predicaba que las mujeres
valen menos. Eso está mal visto. Hoy consiste en algo más soterrado, más sutil.
"Los espacios de trabajo donde solo hay hombres ya son una excepción, o esa
escena del franquismo en la que el hombre llegaba a casa y su esposa le ponía
un coñac", señala Laura Nuño, directora de la cátedra de Género de la

10 „Epoche des europäischen, besonders des deutschen Geisteslebens vom Ende des 18. bis zur Mitte des 19. Jahrhunderts, die in Gegensatz steht zu Aufklärung und Klassik und die geprägt ist durch die Betonung des Gefühls, die Hinwendung zum Irrationalen, Märchenhaften und Volkstümlichen und durch die Rückwendung zur Vergangenheit.“" (Duden 2018)

11 "(of a man): to explain something to a woman in a condescending way that assumes she has no knowledge about the topic" (Merriam-Webster Dictionary 2019); "to explain something to someone in a way that suggests that they are stupid; used especially when a man explains something to a woman that she already understands." (Cambridge Dictionary 2019) 
Universidad Rey Juan Carlos. "Pero los varones siguen manteniendo un espacio de privilegios que reproducen y perpetúan a través de lo que se está llamando neomachismo o micromachismos ${ }^{12}$. (R. Sahuquillo 2017)

El latente feminismo de una protagonista segura de sí misma, con unas metas claras y unos principios inquebrantables por cualquier hombre, está presente desde las primeras líneas, así como la tremenda crítica a las masculinidades que, frente a mujeres con menor capital económico, se comportan de manera condescendiente, sexista y maleducada:

Auch wenn ihr durch den Augenkontakt für einen Moment die Luft weggeblieben war - von einem Typen wie diesem liess sie sich nicht einschüchtern [...] „Stimmt so, Schätzchen”, sagte der Rothaarige und grinste schleimig. Dazu zwinkerte er ihr auch noch anzüglich zu. Emilia kochte innerlich.

Emilia, como vemos, es una mujer de carácter. No le teme al cambio (,Sie probierte städig neue Sorten aus"), factor que puede extrapolarse sin duda a una necesaria evolución feminista de la sociedad. Dedica toda su atención a su trabajo, defendiendo, así, la importancia de la autorrealización profesional frente a la dependencia amorosa. Realiza cuidadosa y apasionadamente, y no quiere ser "una más", una princesa tradicional sin voz ni voto.

Analizando la forma de narrar, resulta destacable cómo Römer plasma los pensamientos de su protagonista, cuyas metas son claras. Emilia no dejará que su enamoramiento desvíe su atención de lo que realmente le importa. De hecho, como al lector, a ella también le repugna la manera en que los millonarios acuden y se comportan en la pastelería: Emilia no tolerará ese tipo de comportamientos.

Por otra parte, la originalidad a la hora de nombrar cada uno de los capítulos de la novela no hace sino acentuarle al lector la importancia del negocio frente al amor. Sin abandonar la temática pastelera, el título de un capítulo será el nombre de un dulce concreto acompañado de una imagen del mismo, lo cual ayuda visualmente al lector a la hora de establecer paralelismos. Y decimos paralelismos porque, bajo cada una de estas imágenes, encontraremos una descripción detallada del pastel ilustrado, de forma que dicha definición supondrá un adelanto del argumento del capítulo que vamos a leer. De esta manera y como ejemplo, el

12 "Micromachismos. Un término que el psicólogo argentino Luis Bonino empezó a utilizar en 1990 para describir un machismo "de baja intensidad", "suave", "cotidiano". Un concepto para referirse a ese más oculto que se ha extendido, pero que disgusta tanto a Nuño como a otras muchas expertas, que sostienen que el término micro minimiza el problema. "A nadie se le ocurriría decir microrracismo, por ejemplo", dice Nuño. "Es machismo, aunque, como estás constantemente expuesta a él en el proceso de socialización, lo naturalizas. Estamos en una cultura sexista, por eso es muy difícil que lo evidencies"." (R. Sahuquillo 20I7) 
primer capítulo, llamado „Samtenes Karamell vereint sich mit der dezenten Säure der Beeren zu einer überraschenden Geschmackexplosion“, narrará el encuentro sorpresivo y emocionante del encuentro chica-chico. Esta metáfora visual supone un juego para el lector, haciendo que, con tan sólo visualizar la primera página del episodio narrado, pueda imaginar lo que presumiblemente ocurrirá en las siguientes hojas, de forma que sus expectativas siempre van a satisfacerse positivamente: si está en lo cierto, habrá sensación de victoria y si, por el contrario, ha errado en su suposición, la sorpresa será mayor.

En cuanto a las descripciones, cabe destacar el detallismo de este subgénero de pastelería: verdaderamente la concreción con la que se presenta este mundo es extraordinaria. El preciosismo destaca desde el primer momento con términos específicos, de forma que, en algún momento, la autora nos hace dudar de si, en realidad, quien ha escrito la novela tiene alguna o mucha relación con la repostería. Una vez más, la imagen del producto pastelero destaca sobre cualquier otra. Asimismo, la genial estructuración de las partes nos introduce de lleno en la historia, recurriendo a una técnica casi cinematográfica, gracias a la que podemos imaginar en nuestra mente lo que está ocurriendo en todo momento.

Con respecto a este último apunte, es de resaltar el detonante de la historia. En toda narración -y en todo guion cinematográfico-, existe un momento de inflexión a partir del que nada volverá a ser lo mismo. No debemos olvidar que se trata de una novela romántica juvenil, con la diferencia de que el tratamiento y la evolución de los personajes desestructuran la tradicional relación de amor y dependencia. Nuestra protagonista, que hasta ahora se encontraba en un estado de reposo, se encuentra, de repente, con un hecho que cambiará su vida para siempre: el encuentro con el chico de la historia. La aparición de los dos hombres de negocios por la puerta es inesperada, acentuando la importancia del momento. La impecable descripción de ambos supone un contraste con respecto a la anterior clienta (Mrs. Witherleaf) y a la propia Emilia, ejemplo de educación y amabilidad, desde el primer momento: „Zwei gekleidete Männer betraten mit hastigen Schritten den Raum". En este caso, la autora utiliza un juego maestro al apelar a los prejuicios del lector: Römer parece conocedora de la psicología humana en general, y juvenil en particular, al presentarnos una imagen tan clara de la personalidad de los dos hombres de negocios mencionando únicamente su aspecto físico y sus actos. A partir de la presentación de estos personajes, el lector sabe perfectamente quién va a ser el chico: la autora juega con nuestros prejuicios, con los clichés sexistas que tan enraizados están en la sociedad y, sin decir quién es "malo" -el que en ningún momento mira a Emilia-y quién es "bueno" -el que se fija en ella, el que ve que es especial, el que la mira, el que se da cuenta de su poder como mujer- lo adivinamos. Sólo con sus actos, con sus actitudes, ya descubrimos al personaje, 
técnica que, de nuevo, recuerda al cine, donde la ausencia de narrador obliga a las imágenes a hablarnos de los caracteres de las personas.

Emilia, a quien no le falta un ápice de inteligencia, amor propio y agudeza visual, desenmascara a los dos enchaquetados en un instante, comparándolos, en su discurso mental:

Der erste, [...] trug einen mürrischen Gesichtsausdruck vor sich her [...]. Emilia hatte schon freundlichere Typen gesehen. [...] Der andere, ein hochgewachsener Mann Mitte dreissig in einem perfekt sitzenden schwarsen Anzug und mit der Aura eines hochrangigen Geschäftsmannes [...]. Sein markantes Kinn war für Emilias Geschmack viel zu glattrasiert, die blauen Augen zu kühl und seine Haare waren so dunkel, dass sie sich fragte, ob das schimmernde Schwarz echt sein konnte. Er war einer dieser typischen New Yorker Businesstypen, stellete Emilia sofort fest.

Rechazando al maleducado compañero de trabajo del joven de ojos azules, Emilia ofrece un mensaje al lector en general y a las jóvenes en particular: nunca escuchar a quienes nos desprecian, a quienes no nos escuchan por el simple hecho de nuestro sexo. Quienes no aceptan que una mujer dirija su propio negocio, resultando como una joven empoderada y con determinación, no merecen nuestro respeto: si no consienten que una mujer sea dueña de sí misma, es porque éste quiere ser su dueño y tiene un ideal de sumisión como relación de amor perfecta.

Seguimos analizando el momento del encuentro para detenernos en la importancia de las miradas. La narración frena de manera evidente en un momento de suma importancia: la primera vez que los protagonistas se miran a los ojos. Y no sólo se miran, sino que se mantienen la mirada, como si de un pulso de dignidades se tratara: Emilia no se amedrenta, nada puede intimidarla. En un momento en el que el joven trata de mostrar su superioridad masculina, Emilia echa por tierra sus principios y rompe con sus esquemas con una simple mirada.

La narración cambia bruscamente de dirección tras finalizar la jornada laboral de Emilia para mostrarnos otra faceta de su personalidad. Es un momento tranquilo, de relajación, en el que la joven recuerda su infancia en Roma y nos transporta a los aromas gastronómicos de su casa en Italia. Lotte Römer utiliza este pasaje como un momento de descanso, una escena donde Emilia comienza a percatarse de la magnitud de la experiencia anterior al descubrir pensamientos y sensaciones cuya mera existencia le provocan un estado de enfado consigo misma. En esta segunda parte del capítulo, conocemos a Violetta, su tía italiana. La breve conversación que ambas tienen otorga mérito al recuerdo de los italoamericanos que migraron buscando trabajo en el Nuevo Mundo, a la vez que glorifica la mezcla de culturas, rechazando la xenofobia: nuevamente, nos encontramos con un alegato a la igualdad. La belleza de lo antiguo se personifica en esta sabia y avispada Tantchen, que no representa sino la faceta subconsciente y las raíces de Emilia. 
Por otra parte, y en cuanto a estética se refiere, cabe analizar la portada de la novela de Römer donde, sobre un fondo rosa, un hombre trajeado del cual sólo vemos su tronco se ajusta una corbata. En primer plano, sin embargo, se encuentran los pasteles: la pasión de Emilia. El conjunto representa una crítica a las rígidas fronteras de género en las que las chicas, sensibles y cursis, "van de rosa" y, los chicos, de azul. En esta imagen se critica precisamente ese cliché absurdo de otorgar un género específico a algo tan abstracto como los colores pues, lo que realmente ve el espectador a primera vista es la repostería que cocina la protagonista: su oficio, su independencia económica, su alegato de libertad como mujer. Por ello no se nos muestra la cara del millonario del fondo: no interesa, no es lo que debería destacar, no es, ni será, la prioridad de Emilia.

En conjunto, Sweet Temptation - Ein Milliardär zum Anbeißen constituye una espléndida novela para desechar de una vez cuestiones aún demasiado presentes en nuestra sociedad como es el machismo, la dependencia emocional y la figura del príncipe azul salvador, de forma que intenta adentrarse en las mentes de quienes leen el relato, personas aún de joven edad, adelantándose a una fosilización de ideas casi imposibles de negar una vez sobrepasan cierta edad.

La autora, sin explicitarlo en ningún momento, deja al sector de lectores al que se dedica la novela, las chicas preadolescentes, un mensaje que, en quienes aún se están formando como personas en valores y experiencias, repercutirá de forma sorprendente. La moraleja que queda tras la lectura es similar a la que los niños de hoy en día adquieren después de ver películas como "Moana", donde ninguna princesa padece dependencia emocional de un valiente príncipe azul para conseguir sus objetivos. Las valientes son ellas, las protagonistas de sus propias historias son ellas.

The change in romantic goals might occur over time as the children born in the last decade begin dating. For many of today's young girls, the first Disney princesses they saw were not Snow White and Cinderella, but Rapunzel and Merida. Soon enough, the Cinderellas and Prince Charmings might become Flynn Riders and Rapunzels. (Batt 2018)

La intencionalidad no deja de ser brillante al no ser explícita y realizarse de una manera sutil y llevadera. $Y$ es que, ante todo, Emilia representa la lucha por los sueños, la humanidad y la confianza en uno mismo, valores que toda persona debería llevar por bandera.

\section{Referencias bibliográficas}

-Bibliografía primaria

Römer, Lotte. Sweet Temptation - Ein Milliardär zum Anbeißen. 2016. 
-Bibliografía secundaria

ADAMED. Glosario de términos psicopatológicos e históricos psiquiátricos. s.f. https://psiquiatria.com/glosario/dependencia-emocional (último acceso: 5 de Enero de 2019).

Antebellum. "The Artifice.» Fairytales and Feminism: "I Don't Wanna be Like Cinderella". 4 de Agosto de 2016. https://the-artifice.com/fairytalesfeminism/ (último acceso: 5 de Enero de 2019).

Batt, Megan. How The Disney Princess Movies Went From Fairy Tales To Feminism. 25 de Abril de 2018. https://studybreaks.com/tvfilm/disney-princess-movies/ (último acceso: 2 de Enero de 2019).

Baum, Antonia. Wie geht Feminismus? 18 de Julio de 2018. https://www.zeit.de/2018/30/das-weibliche-prinzip-meg-wolitzer-metoofeminismus-roman-literatur (último acceso: 8 de Enero de 2019).

$\begin{array}{llll}\text { Cambridge Dictionary. } & \text { Cambdrige } & \text { Dictionary. }\end{array}$ https://dictionary.cambridge.org/es/diccionario/ingles/mansplain (último acceso: 30 de Enero de 2019).

CCOO, Secretaría Confederal de Mujer e Igualdad de. Tribuna Feminista. 18 de Febrero de 2017. https://tribunafeminista.elplural.com/2017/02/quesignifica-el-empoderamiento-de-las-mujeres/ (último acceso: 5 de Enero de 2019).

Clark, Alex. «The Guardian.» The Female Persuasion by Meg Wolitzer review - a timely study of activism. 25 de Julio de 2018. https://www.theguardian.com/books/20I8/jul/25/the-female-persuasionmeg-wolitzer-review (último acceso: 28 de Enero de 2019).

$\begin{array}{llll}\text { Duden. } & \text { Romantik } & \text { Bedeutung. }\end{array}$ https://www.duden.de/rechtschreibung/Romantik\#Bedeutungla (último acceso: 14 de Enero de 2019).

Eckes, Thomas. «Geschlechterstereotype: Von Rollen, Identitäten und Vorurteilen.» En Handbuch Frauen- und Geschlechterforschung, de Thomas Eckes, 12. Freiburg: VS Verlag für Sozialwissenschaften, 2008.

Fariña Busto, María Jesús, y Beatriz Suárez Briones. La crítica literaria feminista, una apuesta por la modernidad. Pontevedra: UNED, s.f.

Franklin, Ruth. «The Atlantic.» The Female Persuasion Should Be a Literary Breakout. Will It Be? Mayo de 2018. https://www.theatlantic.com/magazine/archive/2018/05/the-persuasivefemale/556847/ (último acceso: 22 de Enero de 2019).

Gerhard, Ute. Frauenbewegung und Feminismus: Eine Geschichte seit I789. München: C.H.Beck oHG Verlag, 2009. 
Gualano, Clara. «Clarín, entre mujeres.» Debates actuales: Reflexiones feministas sobre el amor romántico. 16 de Marzo de 2018. https://www.clarin.com/entremujeres/pareja/reflexiones-feministas-amorromantico_0_By27vtStf.html (último acceso: 8 de Enero de 2019).

Merriam-Webster Dictionary. Merriam-Webster. 2019. https://www.merriamwebster.com/dictionary/mansplain (último acceso: 30 de Enero de 2019).

R. Sahuquillo, María. «Micromachismos. Cómo nos explican las cosas los hombres.»
El
País,
27
de
Abril
de
2017.

https://elpais.com/elpais/20I7/04/27/eps/I493244356_I49324.html (último acceso: 4 de Febrero de 2019).

Reisin, Andrej. «Wie hoch ist der Gender Pay Gap wirklich?» Faktenfinder. I5 de Marzo de 2018. https://faktenfinder.tagesschau.de/inland/genderpaygapI03.html (último acceso: 7 de Enero de 2019). 